\title{
Heisenberg spin chain as a worldsheet coordinate for lightcone quantized string
}

\section{Charles B. Thorn}

Institute for Fundamental Theory, Department of Physics, University of Florida, Gainesville, FL 32611, U.S.A.

E-mail: thorn@phys.ufl.edu

ABSTRACT: Although the energy spectrum of the Heisenberg spin chain on a circle defined by

$$
H=\frac{1}{4} \sum_{k=1}^{M}\left(\sigma_{k}^{x} \sigma_{k+1}^{x}+\sigma_{k}^{y} \sigma_{k+1}^{y}+\Delta \sigma_{k}^{z} \sigma_{k+1}^{z}\right)
$$

is well known for any fixed $M$, the boundary conditions vary according to whether $M \in 4 \mathbb{N}+r$, where $r=-1,0,1,2$, and also according to the parity of the number of overturned spins in the state, In string theory all these cases must be allowed because interactions involve a string with $M$ spins breaking into strings with $M_{1}<M$ and $M-M_{1}$ spins (or vice versa). We organize the energy spectrum and degeneracies of $H$ in the case $\Delta=0$ where the system is equivalent to a system of free fermions. In spite of the multiplicity of special cases, in the limit $M \rightarrow \infty$ the spectrum is that of a free compactified worldsheet field. Such a field can be interpreted as a compact transverse string coordinate $x(\sigma) \equiv x(\sigma)+R_{0}$. We construct the bosonization formulas explicitly in all separate cases, and for each sector give the Virasoro conformal generators in both fermionic and bosonic formulations. Furthermore from calculations in the literature for selected classes of excited states, there is strong evidence that the only change for $\Delta \neq 0$ is a change in the compactification radius $R_{0} \rightarrow R_{\Delta}$. As $\Delta \rightarrow-1$ this radius goes to infinity, giving a concrete example of noncompact space emerging from a discrete dynamical system. Finally we apply our work to construct the three string vertex implied by a string whose bosonic coordinates emerge from this mechanism.

Keywords: Conformal Field Models in String Theory, Bethe Ansatz, Matrix Models

ArXiv ePrint: 2009.13419 


\section{Contents}

1 Introduction 1

2 Overlaps in free fermion systems 4

3 Heisenberg spin system $\quad 6$

3.1 Hamiltonian in fermionic language 6

$3.2 \Delta=0$ : free fermion case $\quad 7$

3.3 Dependence of ground energy on the nature of $M \quad 7$

$\begin{array}{lr}3.4 \text { Energy eigenstates for } \Delta=0 & 9\end{array}$

$\begin{array}{lll}3.5 & \text { Energy levels and degeneracies for } M \text { large } & 10\end{array}$

4 Conformal invariance and bosonization at large $M \quad 13$

$\begin{array}{lll}4.1 & \text { Virasoro generators } & 13\end{array}$

$\begin{array}{ll}4.2 \text { Bosonization of the spin system at } \Delta=0 & 16\end{array}$

$\begin{array}{lll}4.3 & \text { Interpretation of zero mode operators } & 18\end{array}$

5 Spin chain contribution to 3 string vertex 18

$\begin{array}{lll}5.1 \text { Preliminary discussion } & 18\end{array}$

$\begin{array}{lll}5.2 & \text { Three string overlap } & 19\end{array}$

\section{Introduction}

This article is a technical follow up to [1], which proposed a string bit model $[2,3]$ for superstring [4-9] in which space is not fundamental, but is rather an emergent feature of the string bit dynamics. Briefly, a string bit ${ }^{1}$ is described by annihilation (and creation) operators, which are $N \times N$ matrices $\left(\phi_{\left[a_{1}, \ldots a_{n}\right]}^{A}\right)_{\alpha}^{\beta}\left(\left(\phi_{\left[a_{1}, \ldots a_{n}\right]}^{A}\right)_{\alpha}^{\beta \dagger}\right)$, where $\alpha, \beta=1, \ldots, N$ are the matrix indices, $a_{1}, \cdots, a_{n}$ are spinor indices each taking values $1, \ldots, s$, with $n$ ranging from 0 to $s$. The $\phi$ are completely antisymmetric in these spinor indices. The index $A$ labels additional string bit internal states in some models.

In these models the emergence of space starts with the emergence of a worldsheet in 't Hooft's limit $N \rightarrow \infty$ [10]. In this limit the bits form into noninteracting closed chains. The lowest energy chains have an infinite number of bits in certain models [11-13]: then low energy chains look like strings moving in one space dimension. This one spatial dimension emerges as the conjugate to bit number $M$, which for $M \rightarrow \infty$ can be treated as a positive continuous variable and interpreted as the lightcone $[14,15]$ longitudinal momentum $P^{+} / m$, where $m$ is the $P^{+}$carried by each string bit. This emergent coordinate is therefore appropriately named $x^{-}$.

\footnotetext{
${ }^{1}$ Since it is a quantum entity with a finite number of states, it might be thought of as a $q$-byte.
} 
However, instead of transverse spatial dimensions, fluctuations in the string bit internal states, labeled by the $a$ and $A$ indices, become fermionic or compact bosonic worldsheet fields. For example, the spinor indices on the string bit operator are promoted, on large bit number chains [16] to $s$ worldsheet Fermi fields of the Green-Schwarz type [17, 18]. If the label $A$ is absent, the quantum overlap between a two string state and a one string state is consistent with Lorentz covariance $(\mathrm{SO}(1,1))$ only if $s=24[19,20]$. I call this string in 1 space 1 time dimensions the protostring. It has an interesting degree of freedom count: 16 of the 24 Grassmann dimensions can be bosonized to 8 compactified transverse boson dimensions leaving 8 Grassmann dimensions. This is the worldsheet field content of the superstring. Of course the familiar super Poincaré covariant superstring requires 8 noncompact transverse dimensions in addition to an operator applied to the overlap at the string separation point.

One way for noncompact dimensions to arise from fluctuations of a finite valued index is to appeal to the one dimensional Heisenberg spin chain. I noted in [1] that the degrees of freedom labeled by $A$ could be engineered so that the 't Hooft $N \rightarrow \infty$ limit produces an additional worldsheet subsystem describing a Heisenberg spin chain with Hamiltonian

$$
H=\frac{1}{4} \sum_{k=1}^{M}\left(\sigma_{k}^{x} \sigma_{k+1}^{x}+\sigma_{k}^{y} \sigma_{k+1}^{y}+\Delta \sigma_{k}^{z} \sigma_{k+1}^{z}\right)
$$

where $M$ is the number of bits in the chain, In fact one can design the string bit Hamiltonian to produce several independent Heisenberg spin chains. Although the notation suggests that the $\sigma$ 's refer to physical spin, we shall refer to the eigenvalues of $\sigma^{z}$ as "charge". This Hamiltonian has been very well studied. Eigenstates of $H$ (probably all of them) can be found via the Bethe ansatz [21], which exploits the fact that the total charge $Q=\sum_{k=1}^{M} \sigma_{k}^{z}$ commutes with $H$, so that an eigenstate of $H$ may be assumed to have a fixed charge given by $Q=M-2 q$ where $q$ is the number of overturned spins compared to a reference state with all spins up. Yang and Yang [22, 23] solved the Bethe ansatz equations for the ground state in each charge sector, in the limit $M \rightarrow \infty$. For $\Delta$ in the range $-1<\Delta<1$, the energy excitations were shown to be of order $O\left(M^{-1}\right)$ corresponding to a nontrivial continuum"stringy" limit. Earlier Lieb, Schultz, and Mattis had solved the $\Delta=0$ case exactly [24], based on the fact that the Heisenberg spin system is then equivalent to a system of free fermions. A discussion of the eigenstates (with $E=O\left(M^{-1}\right.$ ) relevant to the interpretation of the system as a compactified worldsheet coordinate is given in $[1,25]$. The compactification radius $R$ depends on $\Delta$ :

$$
R_{\Delta}^{2}=\frac{\pi \alpha^{\prime}}{\mu}, \quad \Delta=-\cos \mu .
$$

Here the Regge slope parameter $\alpha^{\prime}=1 /\left(2 \pi T_{0}\right)$, where $T_{0}$ is the rest tension of the string determined by the excitation energy spectrum. This interpretation is solid when $\Delta=0$, but for $\Delta \neq 0$ it relies on the Bethe ansatz and the calculations in [22,23] for some selected classes of excitation. These included the lowest states with any fixed $Q$ and $\hat{P} / \pi$ and spin wave excitations relative to these lowest states. Here $\hat{P}=P-\pi q$ with $P$ the total lattice momentum modulo $O\left(M^{-1}\right)$ as $M \rightarrow \infty$. For $\Delta \neq 0$ it is logically possible that the Bethe 
ansatz misses some eigenstates, which could also be missed by the compactified coordinate interpretation.

The equivalence of the Heisenberg spin system to a compactified bosonic worldsheet field when $-1<\Delta<1$ provides a shortcut to the calculation of string scattering amplitudes: the string technology for such worldsheet systems is very well developed. However there remains some doubt that the bosonization formalism really captures all aspects of the Heisenberg system at large $M$. The purpose of this article is to flesh out the connection between the original spin system and its bosonized counterpart. It may be that resort to numerical methods may be needed. So we lay out in painstaking detail the Fermi Bose connection at the free fermion point $\Delta=0$. This is to test the two descriptions of the string interactions.

This work is quite tedious because the worldsheet fermionic fields of the system have different boundary conditions for different values of the charge $Q$. For example, assuming that $M$ is a multiple of 4 , the fermionic fields are antiperiodic when $Q$ is also a multiple of 4 , but periodic when $Q$ is an odd multiple of 2 . This assignment is reversed if $M$ is an odd multiple of 2 . When $M$ is odd the situation is even more involved. In string theory the interactions change the number of bits in a string by breaking one string into two or joining two strings to form 1 . Since bit number is conserved, the three strings in the process cannot have the same number of bits. One must allow strings with any number of bits. Thus one must do a separate calculation for 8 different sectors for each of the three chains participating in the vertex. We set up the vertex construction in a way to cover any of these cases,

In spite of all the different separate cases, in the limit $M \rightarrow \infty$ the final result for the spectrum of the Heisenberg Hamiltonian at the free fermion point is simply described in the language of bosonization: the energy spectrum is identical to that of a bosonic worldsheet field compactified on a circle of a specific radius $R_{0}$ [25]. Thus the contribution to the string vertex at $\Delta=0$ can, in principle, be evaluated in two ways: either in the free fermion language, or the free boson language. Whether these two methods give identical results is not clear. Unfortunately we are so far unable to obtain the $M \rightarrow \infty$ limit for the fermionic version for reasons we shall explain. But since bosonization is only valid in this limit, a direct comparison cannot yet be made.

This bosonized language makes the $\Delta \neq 0$ case tractable, For the energy spectrum and degeneracies for $M$ large then includes that of a boson compactified on a circle of different radius $R_{\Delta}$. Interestingly there is a limit $(\Delta \rightarrow-1)$ in which $R \rightarrow \infty$, yielding an uncompactified coordinate, which can be identified as one of the transverse coordinates of a string moving in flat space. For the superstring we would need 8 such Heisenberg systems along with the 8 Fermi Grassmann dimensions that emerge from fluctuations in the spinor indices. While the fermionic Hamiltonian has a quartic interaction term proportional to $\Delta$, the bosonic form of the Hamiltonian is quadratic for all $\Delta$, and we can calculate the overlap using the methods of Mandelstam [26-28], Indeed, for the decompactification limit Mandelstam's three string vertex is the desired answer.

In section 2 we set up overlap calculations of the sort required in string theory for general free fermion systems. In sections 3 and 4 we do the painstaking, case by case, work 
of enumerating the (already known) energy eigenstates and eigenvalues for the Heisenberg spin system at $\Delta=0$. We summarize all the separate cases for ground states in a table. Then we describe the complete spectrum and degeneracies in the large $M$ limit, using partition functions. In section 4 we further characterize the large $M$ limit by constructing the Virasoro generators and the operator bosonization formulas. Finally in section 5 we apply the results of section 2 to construct the overlap at $\Delta=0$ for finite $M$, in terms of an $M \times M$ matrix. A submatrix of this matrix must be inverted to obtain the overlap. This can of course be done numerically for finite $M$. We give the large $M$ limit of this matrix, and explain why Goldstone's method (at least in its original form) for inverting the matrix, which succeeds for the bosonic string and Green-Schwarz superstring overlap [29], falls short. Nonetheless, we can see from the limit that left and right moving spin waves do decouple from each other, as they should. This feature is shared by the overlap in bosonic language. The latter can be extended to $\Delta \neq 0$, allowing Mandelstam's methods to be applied to the general case.

\section{Overlaps in free fermion systems}

We are interested in systems that undergo a sudden change of Hamiltonian at various times, as occurs in the worldsheet description of string theory. But here we concentrate on just a single sudden change in a system of fermions described by anticommuting energy annihilation and creation operators. So for the first (second) Hamiltonian we introduce annihilation operators $B_{k}\left(b_{k}\right)$ and creation operators $B_{k}^{\dagger}\left(b_{k}^{\dagger}\right)$ :

$$
\begin{array}{rlrl}
{\left[H_{1}, B_{k}\right]} & =-\Omega_{k} B_{k}, & \left\{B_{k}, B_{l}^{\dagger}\right\} & =\delta_{k l} \\
{\left[H_{2}, b_{k}\right]} & =-\omega_{k} b_{k}, \quad\left\{b_{k}, b_{l}^{\dagger}\right\}=\delta_{k l}
\end{array}
$$

We shall not assume that the energies $\omega, \Omega$ are positive. So, as in the Dirac system, the ground states have negative energy levels occupied. We shall assume there is a common reference eigenstate $|0\rangle$ annihilated by both $B_{k}$ and $b_{k}$, as well as by $H_{1}$ and $H_{2}$. (This is a feature of the Heisenberg spin system at the free fermion point, and it's also a feature of the Dirac free fermion system.) Then any eigenstate of $H_{1}$ or $H_{2}$ can be obtained from the coherent states

$$
\begin{aligned}
& |\gamma\rangle=e^{\sum_{s} \gamma_{s} B_{s}^{\dagger}}|0\rangle \\
& |\alpha\rangle=e^{\sum_{r} \alpha_{r} b_{r}^{\dagger}}|0\rangle
\end{aligned}
$$

where $\alpha, \gamma$ are Grassmann numbers. Let $G$ be the set of $s$ for which $\Omega_{s}<0$ and $g$ be the set of $r$ for which $\omega_{r}<0$. Then the ground states can be expressed as

$$
\begin{aligned}
|G\rangle & =\left.\prod_{s \in G} \frac{d}{d \gamma_{s}}|\gamma\rangle\right|_{\gamma=0}=\prod_{s \in G} B_{s}^{\dagger}|0\rangle \\
|g\rangle & =\left.\prod_{r \in g} \frac{d}{d \alpha_{r}}|\alpha\rangle\right|_{\alpha=0}=\prod_{r \in g} b_{r}^{\dagger}|0\rangle
\end{aligned}
$$


The ordering of the operators applied to a ket is set by convention to be increasing index from left to right. This ordering is reversed in $\langle G|,\langle g|$. The bra coherent states are written

$$
\begin{aligned}
& \langle\gamma|=\langle 0| e^{\sum_{s} \gamma_{s} B_{s}} \\
& \langle\alpha|=\langle 0| e^{\sum_{r} \alpha_{r} b_{r}}
\end{aligned}
$$

so in obtaining $\langle G|$ and $\langle g|$ from the coherent states one just reverses the ordering of the derivatives relative to the ket construction. The overlaps of interest to us are between states obtained by applying monomials of positive energy creation operators and negative energy annihilation operators to $|G\rangle$ or $|g\rangle$. In terms of coherent states

$$
\begin{aligned}
& |\gamma, \hat{\gamma}\rangle=e^{\sum_{s \notin G} \gamma_{s} B_{s}^{\dagger}} e^{\sum_{s \in G} \hat{\gamma}_{s} B_{s}}|G\rangle \\
& |\alpha, \hat{\alpha}\rangle=e^{\sum_{r \notin g} \alpha_{s} b_{r}^{\dagger}} e^{\sum_{r \in g} \hat{\alpha}_{r} b_{r}}|g\rangle
\end{aligned}
$$

The annihilation operators create holes in the negative energy sea.

We are interested in matrix elements

$$
\left\langle g\left|e^{\sum_{r \in g} \hat{\alpha}_{r} b_{r}^{\dagger}} e^{\sum_{r \notin g} \alpha_{r} b_{r}} e^{\sum_{s \notin G} \gamma_{s} B_{s}^{\dagger}} e^{\sum_{s \in G} \hat{\gamma}_{s} B_{s}}\right| G\right\rangle
$$

which can be computed by taking derivatives of

$$
\begin{aligned}
& \left\langle 0\left|e^{\sum_{r \in g} \alpha_{r} b_{r}} e^{\sum_{r \in g} \hat{\alpha}_{r} b_{r}^{\dagger}} e^{\sum_{r \notin g} \alpha_{r} b_{r}} e^{\sum_{s \notin G} \gamma_{s} B_{s}^{\dagger}} e^{\sum_{s \in G} \hat{\gamma}_{s} B_{s}} e^{\sum_{s \in G} \gamma_{s} B_{s}^{\dagger}}\right| 0\right\rangle \\
& =e^{\sum_{r \in g} \hat{\alpha}_{r} \alpha_{r}+\sum_{s \in G} \gamma_{s} \hat{\gamma}_{s}}\left\langle 0\left|e^{\sum_{r} \alpha_{r} b_{r}} e^{\sum_{s} \gamma_{s} B_{s}^{\dagger}}\right| 0\right\rangle \\
& =e^{\sum_{r \in g} \hat{\alpha}_{r} \alpha_{r}+\sum_{s \in G} \gamma_{s} \hat{\gamma}_{s}}\left\langle 0\left|e^{\sum_{s} \gamma_{s} \alpha_{r}\left\{b_{r} \cdot B_{s}^{\dagger}\right\}}\right| 0\right\rangle=e^{\sum_{r \in g} \hat{\alpha}_{r} \alpha_{r}+\sum_{s \in G} \gamma_{s} \hat{\gamma}_{s}+\sum_{s} \gamma_{s} \alpha_{r} D_{s r}}
\end{aligned}
$$

In these fermion models $\left\{b_{r} . B_{s}^{\dagger}\right\}=D_{s r}$ is a numerical matrix. This matrix also gives the relation between the two bases:

$$
B_{s}^{\dagger}=D_{s r} b_{r}^{\dagger}, \quad B_{s}=D_{s r}^{*} b_{r}=b_{r} D_{r s}^{\dagger}
$$

The anticommutation relations imply that $D$ is a unitary matrix. If we think of $B, b$ as row vectors and $B^{\dagger}, b^{\dagger}$ as column vectors, one can safely suppress indices in some equations, e.g.

$$
B^{\dagger}=D b^{\dagger}, \quad B=b D^{\dagger}, \quad b^{\dagger}=D^{\dagger} B^{\dagger}, \quad b=B D .
$$

To construct the ket $|G\rangle$ we simply apply a product of derivatives w.r.t. $\gamma_{s}$ with $s \in G$ after which we set these $\gamma$ to zero.

$$
\begin{gathered}
\left\langle 0\left|e^{\sum_{r \in g} \alpha_{r} b_{r}} e^{\sum_{r \in g} \hat{\alpha}_{r} b_{r}^{\dagger}} e^{\sum_{r \notin g} \alpha_{r} b_{r}} e^{\sum_{s \notin G} \gamma_{s} B_{s}^{\dagger}} e^{\sum_{s \in G} \hat{\gamma}_{s} B_{s}}\right| G\right\rangle \\
=e^{\sum_{r \in g} \hat{\alpha}_{r} \alpha_{r}+\sum_{s \notin G} \gamma_{s} \alpha_{r} D_{s r}} \prod_{s \in G}\left(\hat{\gamma}_{s}+\sum_{r} \alpha_{r} D_{s r}\right)
\end{gathered}
$$

Notice that this procedure gives identical results to simply integrating (2.11) over $\gamma_{s}$ with $s \in G$. Similarly the bra $\langle g|$ is obtained by integrating (2.11) over $\alpha_{r}$ with $r \in g$. 
Now define $D^{G g}$ to be the matrix formed by restricting the matrix $D_{s r}$ to $r \in g$ and $s \in G$. We shall slso assume that the sets $G, g$ are the same size, so that $D^{G g}$ is a square matrix, Then integrating (2.11) over both $\gamma_{s}$ with $s \in G$ and $\alpha_{r}$ with $r \in g$ leads to the desired formula

$$
\begin{aligned}
& \left\langle g\left|e^{\sum_{r \in g} \hat{\alpha}_{r} b_{r}^{\dagger}} e^{\sum_{r \notin g} \alpha_{r} b_{r}} e^{\sum_{s \notin G} \gamma_{s} B_{s}^{\dagger}} e^{\sum_{s \in G} \hat{\gamma}_{s} B_{s}}\right| G\right\rangle \\
& =\operatorname{det} D^{G g} \exp \left[\sum_{s \notin G, r \notin g} \gamma_{s} \alpha_{r} D_{s r}\right. \\
& \left.\quad+\sum_{t \in g, u \in G}\left(\hat{\gamma}_{u}+\sum_{r \notin g} \alpha_{r} D_{u r}\right)\left(D^{G g}\right)_{t u}^{-1}\left(\hat{\alpha}_{t}+\sum_{s \notin G} \gamma_{s} D_{s t}\right)\right]
\end{aligned}
$$

This formula gives all of the overlaps of interest in terms of the matrix $D_{r s}{ }^{2}$

\section{Heisenberg spin system}

\subsection{Hamiltonian in fermionic language}

We write the Hamiltonian for the Heisenberg one dimensional spin system as a nominally antiferromagnetic system with $M$ spins

$$
H=\frac{1}{4} \sum_{k=1}^{M}\left(\sigma_{k}^{x} \sigma_{k+1}^{x}+\sigma_{k}^{y} \sigma_{k+1}^{y}+\Delta \sigma_{k}^{z} \sigma_{k+1}^{z}\right)
$$

and impose periodic boundary conditions $\sigma_{M+1} \equiv \sigma_{1}$. The $\sigma_{k}$ 's are Pauli spin matrices independent on each site. They satisfy the commutator algebra

$$
\left[\sigma_{k}^{a}, \sigma_{l}^{b}\right]=0, \quad l \neq k ; \quad\left\{\sigma_{k}^{a}, \sigma_{k}^{b}\right\}=2 \delta_{a b} .
$$

While the notation suggests the spin interpretation of the Pauli matrices, they could easily represent an internal symmetry like isospin. That's how we regard them in the string bit models. An important symmetry of the Heisenberg model is generated by the charge $Q=\sum_{k} \sigma_{k}^{z}$, which commutes with $H$ because rotational symmetry about the $z$ axis is unbroken by $\Delta \neq 0$. This symmetry underlies the success of the Bethe ansatz.

The Jordan-Wigner trick defines anticommuting variables $S_{k}^{a}, a=x, y$ by

$$
S_{k}^{x, y}=\sigma_{k}^{x, y} \prod_{l=1}^{k-1} \sigma_{l}^{z},
$$

so that

$$
\left\{S_{k}^{a}, S_{l}^{b}\right\}=2 \delta_{k l} \delta_{a b}, \quad a, b \neq z .
$$

Using $\sigma_{k}^{a} \sigma_{k}^{b}=\delta_{a b}+i \epsilon_{a b c} \sigma_{k}^{c}$ one can express $H$ entirely in terms of the $S$ 's. Then the $\Delta$ term is quartic in the $S$ 's, so the free fermion case corresponds to $\Delta=0$ which we assume in the rest of this section and all of the next section.

\footnotetext{
${ }^{2}$ I thank the referee for reminding me of the well-known equivalence between Grassmann integration and differentiation in the context of simplifying the derivation of (2.14).
} 


\section{2 $\Delta=0:$ free fermion case}

The Hamiltonian then becomes

$$
H=\frac{i}{4} \sum_{k=1}^{M-1}\left(S_{k}^{x} S_{k+1}^{y}-S_{k}^{y} S_{k+1}^{x}\right)-\frac{i}{4}\left(S_{M}^{x} S_{1}^{y}-S_{M}^{y} S_{1}^{x}\right) \Omega
$$

where $\Omega=\prod_{k=1}^{M} \sigma_{k}^{z}$. We find it convenient to use raising and lowering operators $S_{k}=\left(S_{k}^{x}+i S_{k}^{y}\right) / 2$ and $S_{k}^{\dagger}$, satisfying $\left\{S_{k}, S_{l}\right\}=0$ and $\left\{S_{k}, S_{l}^{\dagger}\right\}=\delta_{k l}$. Then $H$ reads

$$
H=\frac{1}{2} \sum_{k=1}^{M-1}\left(S_{k}^{\dagger} S_{k+1}+S_{k+1}^{\dagger} S_{k}\right)-\frac{1}{2}\left(S_{M}^{\dagger} S_{1}+S_{1}^{\dagger} S_{M}\right) \Omega .
$$

Clearly the boundary conditions depend on the value of $\Omega= \pm 1$. The energy raising and lowering operators are just Fourier transforms of the $S$ 's.

$$
S_{k}=\frac{1}{\sqrt{M}} \sum_{r} e^{2 \pi i k r / M} B_{r}, \quad B_{r}=\frac{1}{\sqrt{M}} \sum_{k} e^{-2 \pi i k r / M} S_{k}
$$

Here $r=n+\alpha$, with $n$ an integer in $0 \leq n \leq M-1$. The fractional part of $r$ determines the boundary conditions on $S_{k}$ :

$$
S_{M+1}=e^{2 \pi i \alpha} S_{1}
$$

Thus $\alpha=0$ for $\Omega=-1$ and $\alpha=1 / 2$ for $\Omega=+1$. In other words $r$ ranges through integers (half odd integers) if $\Omega=-1(\Omega=+1)$. Plugging the expansions for $S, S^{\dagger}$ into $H$ gives

$$
H=\sum_{r} \cos \frac{2 \pi r}{M} B_{r}^{\dagger} B_{r}, \quad\left\{B_{r} \cdot B_{s}^{\dagger}\right\}=\delta_{r s}
$$

Thus $B_{r}^{\dagger}$ creates an amount of energy $\cos (2 \pi r / M)$, which is negative for $r$ in the range $M / 4<r<3 M / 4$. In some cases $r$ can be exactly $M / 4$ or $3 M / 4$, in which case the corresponding $B^{\dagger}$ creates zero energy. In constructing the ground state, all negative energy levels usually must be occupied, ${ }^{3}$ but the existence of zero energy modes means the ground level is degenerate, and the selection of ground state is ambiguous, depending on how many of the zero energy levels are filled. The nonzero energy levels with $r \neq M / 2$ are doubly degenerate due to the symmetry under $r \rightarrow M-r$.

\subsection{Dependence of ground energy on the nature of $M$}

The Heisenberg spin chain is antiferromagnetic in character for all values $-1<\Delta<+1$. This means that the ground state tends to minimize $|Q|$. Starting with the state with all spins up $(Q=M)$, by overturning $q$ of the spins the charge is lowered to $Q=M-2 q$. When $M$ is even, the ground state has $Q=0$ because one can overturn exactly half of the spins in the state $|0\rangle$. However when $M$ is odd, this possibility is frustrated and the ground state has $Q= \pm 1$. But in each of these cases there are two subcases.

\footnotetext{
${ }^{3}$ Because for fixed $\Omega$ excited states always involve at least two levels, it is possible that the lowest energy state involves an unoccupied negative energy level or an occupied positive energy level, This happens in the spin system when $M$ is odd.
} 


\begin{tabular}{|c|c|c|c|c|c|c|}
\hline $\mathrm{M}$ & $\mathrm{G}$ & $\mathrm{q}$ & $Q$ & $\frac{\hat{P}}{\pi}$ & $\Omega$ & $E_{G}$ \\
\hline$M \in 4 \mathbb{N}$ & $\frac{M+2}{4}, \cdots, \frac{3 M-2}{4} \in \frac{1}{2}+\mathbb{Z}$ & $\frac{M}{2}$, even & 0 & 0 & +1 & $-\csc \frac{\pi}{M}$ \\
\hline & $\frac{M+4}{4}, \cdots, \frac{3 M-4}{4} \in \mathbb{Z}$ & $\frac{M-2}{2}$, odd & +2 & 0 & -1 & $-\cot \frac{\pi}{M}$ \\
\hline & $\frac{M}{4}, \cdots, \frac{3 M}{4} \in \mathbb{Z}$ & $\frac{M+2}{2}$, odd & -2 & 0 & -1 & $-\cot \frac{\pi}{M}$ \\
\hline$M \in 4 \mathbb{N}+2$ & $\frac{M+2}{4}, \cdots, \frac{3 M-2}{4} \in \mathbb{Z}$ & $\frac{M}{2}$, odd & 0 & 0 & -1 & $-\csc \frac{\pi}{M}$ \\
\hline & $\frac{M+4}{4}, \cdots, \frac{3 M-4}{4} \in \frac{1}{2}+\mathbb{Z}$ & $\frac{M-2}{2}$, even & +2 & 0 & +1 & $-\cot \frac{\pi}{M}$ \\
\hline & $\frac{M}{4}, \cdots, \frac{3 M}{4} \in \frac{1}{2}+\mathbb{Z}$ & $\frac{M+2}{2}$, even & -2 & 0 & +1 & $-\cot \frac{\pi}{M}$ \\
\hline$M \in 4 \mathbb{N}+1$ & $\frac{M+1}{4}, \cdots, \frac{3 M-5}{4} \in \frac{1}{2}+\mathbb{Z}$ & $\frac{M-1}{2}$, even & +1 & $-\frac{q}{M}$ & +1 & $-\cot \frac{\pi}{M} \cos \frac{\pi}{2 M}$ \\
\hline & $\frac{M+5}{4}, \cdots, \frac{3 M-1}{4} \in \frac{1}{2}+\mathbb{Z}$ & $\frac{M-1}{2}$, even & +1 & $\frac{q}{M}$ & +1 & $-\cot \frac{\pi}{M} \cos \frac{\pi}{2 M}$ \\
\hline & $\frac{M+3}{4}, \cdots, \frac{3 M+1}{4} \in \mathbb{Z}$ & $\frac{M+1}{2}$, odd & -1 & $\frac{q}{M}$ & -1 & $-\cot \frac{\pi}{M} \cos \frac{\pi}{2 M}$ \\
\hline & $\frac{M-1}{4}, \cdots, \frac{3 M-3}{4} \in \mathbb{Z}$ & $\frac{M+1}{2}$, odd & -1 & $-\frac{q}{M}$ & -1 & $-\cot \frac{\pi}{M} \cos \frac{\pi}{2 M}$ \\
\hline$M \in 4 \mathbb{N}-1$ & $\frac{M+1}{4}, \cdots, \frac{3 M-5}{4} \in \mathbb{Z}$ & $\frac{M-1}{2}$, odd & +1 & $-\frac{q}{M}$ & -1 & $-\cot \frac{\pi}{M} \cos \frac{\pi}{2 M}$ \\
\hline & $\frac{M+5}{4}, \cdots, \frac{3 M-1}{4} \in \mathbb{Z}$ & $\frac{M-1}{2}$, odd & +1 & $\frac{q}{M}$ & -1 & $-\cot \frac{\pi}{M} \cos \frac{\pi}{2 M}$ \\
\hline & $\frac{M+3}{4}, \cdots, \frac{3 M+1}{4} \in \frac{1}{2}+\mathbb{Z}$ & $\frac{M+1}{2}$, even & -1 & $\frac{q}{M}$ & +1 & $-\cot \frac{\pi}{M} \cos \frac{\pi}{2 M}$ \\
\hline & $\frac{M-1}{4}, \cdots, \frac{3 M-3}{4} \in \frac{1}{2}+\mathbb{Z}$ & $\frac{M+1}{2}$, even & -1 & $-\frac{q}{M}$ & +1 & $-\cot \frac{\pi}{M} \cos \frac{\pi}{2 M}$ \\
\hline
\end{tabular}

Table 1. Momentum modes contributing to the ground state(s) in both $\Omega= \pm 1$ sectors for fixed $M$, together with values of $q, Q, \hat{P} / \pi$, and energy in each case.

For $M$ even they are $M=$ a multiple of 4 , with an even number of negative energy states filled with half odd integer modes; and $M=2+$ a multiple of 4 , with an odd number of negative energy states filled with integer modes. The table shows that when the mode numbers in these two cases are expressed in terms of $M$, the patterns in the two cases are identical and in particular the energies are identical functions of $M$. The same story holds for the lowest energy states with opposite value of $\Omega$ to the ground state. The degeneracy between $Q=\Sigma= \pm 2$ is due to time reversal invariance. It can also be seen by applying the operator $B_{M / 4}^{\dagger} B_{3 M / 4}^{\dagger}$, which creates 0 energy and destroys 4 units of charge, to the $Q=+2$ ground state.

When $M$ is odd we again have two cases for which $M$ is \pm 1 plus a multiple of 4 . The $\Omega$ 's are opposites, but energy patterns are identical when expressed in terms of $M$. For a fixed $M$ there are 4 degenerate states, two with $Q=+1$ and two with $Q=-1$. Again the degeneracy of the $Q= \pm 1$ states is a consequence of time reversal, but since they have opposite $\Omega$ 's there is no simple operator linking them as in the even case. But the two states with the same charge are linked by simple operators: $B_{(3 M-1) / 4}^{\dagger} B_{(M+1) / 4}$ takes line 7 to line 8 (or line 11 to line 12 ) in the table, while $B_{(M-1) / 4}^{\dagger} B_{(3 M+1) / 4}$ takes line 9 to line 10 (or line 13 to line 14). Both of these operators create 0 energy, because they create and destroy two states of the same energy:

$$
\cos \frac{2 \pi(3 M-1)}{4 M}=-\sin \frac{\pi}{2 M}=\cos \frac{2 \pi(M+1)}{4 M} .
$$




\subsection{Energy eigenstates for $\Delta=0$}

The description of the complete set of energy eigenstates is complicated by the need to switch mode numbers from half odd integers to integers when $\Omega$ changes from +1 to -1 . The ground states delineated in the previous subsection can form convenient starting eigenstates. For fixed $M$ one must pick a ground state in each of the two $\Omega$ sectors. Then a complete set of states is generated by applying even monomials of eigenoperators, moded appropriately, to each of the choices. When there is a degeneracy of ground states in a given sector one can pick one of them at will. For definiteness, we choose lines 1,2,4,5,7,9,11,and 13 as our ground states. Then the even monomials will consist of half odd integer moded eigenoperators for cases 1,5,7,13 and integer moded eigenoperators for cases 2,4,9,11.

The momentum carried by a mode $r$ is $p_{r}=2 \pi r / M$. This relation is used to calculate the entries in the table under $\hat{P} / \pi . \hat{P} \equiv P-\pi q$, where $\mathrm{P}$ is the total momentum of the system. It commutes with the Hamiltonian because there is a discrete lattice translational invariance with periodic boundary conditions. At the level of individual modes the definition of $\hat{P}$ corresponds to $\hat{p}_{r} \equiv p_{r}-\pi$. The $\hat{p}_{r}$ distribution is centered around 0 instead of around $\pi$. The eigenvalue of $\hat{P}$ distinguishes the degenerate ground states with equal energy and charge.

One can construct any excited state by applying combinations of bilinears of creation operators to the ground state in a given sector. For this purpose, creation operators are $B_{r}^{\dagger}$ for $r \notin G$ and $B_{s}$ for $s \in G$. then the possible bilinears are:

$$
B_{r}^{\dagger} B_{s}^{\dagger}, \quad B_{r}^{\dagger} B_{t}, \quad B_{t} B_{u}, \quad r, s \notin G ; \quad t, u \in G
$$

If two or more bilinears are applied, the state will be nonzero only if all indices are different. Furthermore two nonzero states are proportional if the sets of indices are permutations of each other. The listed bilinears change $Q$ by $-4,0,+4$ respectively; $\hat{P}$ by $2 \pi[-1+(r+s) / M]$, $2 \pi(r-t) / M,-2 \pi[-1+(t+u) / M]$ respectively; and $E$ by $\cos (2 \pi r / M)+\cos (2 \pi s / M)$, $\cos (2 \pi r / M)-\cos (2 \pi t / M),-\cos (2 \pi t / M)-\cos (2 \pi u / M)$ respectively.

To get stringy physics, we should take $M \rightarrow \infty$, and concentrate on low energy excitations. These occur when the momentum indices stay a finite distance from $M / 4$ or from $3 M / 4$ in the limit. The energies created by $B_{r}^{\dagger}$ are

$$
\begin{aligned}
& \cos \frac{2 \pi r}{M}=\cos \left(\frac{\pi}{2}+\frac{2 \pi(r-M / 4)}{M}\right)=-\sin \frac{2 \pi(r-M / 4)}{M} \sim-\frac{2 \pi(r-M / 4)}{M} \\
& \cos \frac{2 \pi s}{M}=\cos \left(\frac{3 \pi}{2}+\frac{2 \pi(s-3 M / 4)}{M}\right)=\sin \frac{2 \pi(s-M / 4)}{M} \sim \frac{2 \pi(s-3 M / 4)}{M}
\end{aligned}
$$

Notice that in the limit the excitation energies are plus or minus the momenta relative to the appropriate Fermi momentum $M / 4$ or $3 M / 4$. Also keep in mind that $r$ must be chosen half odd or integer according to whether $\Omega$ of the state is positive or negative. Write the excitation energies as $2 \pi \lambda_{r} / M$ we see that $\lambda_{r}$ is integer or half odd integer when $M$ is even, but is $\pm 1 / 4+$ integer when $M$ is odd. 
The large $M$ behaviors of the ground energies (last column of the table) are given by:

$$
\begin{aligned}
-\csc \frac{\pi}{M} & \sim-\frac{M}{\pi}\left[1+\frac{\pi^{2}}{6 M^{2}}+O\left(M^{-4}\right)\right]=-\frac{M}{\pi}-\frac{\pi}{6 M}+O\left(M^{-3}\right) \\
-\cot \frac{\pi}{M} & \sim-\frac{M}{\pi}-\frac{\pi}{6 M}+\frac{\pi}{2 M}+O\left(M^{-3}\right) \\
-\cot \frac{\pi}{M} \cos \frac{\pi}{2 M} & \sim-\frac{M}{\pi}-\frac{\pi}{6 M}+\frac{\pi}{2 M}+\frac{\pi}{8 M}+O\left(M^{-3}\right)
\end{aligned}
$$

Inspection shows that the right sides of these equations can be summarized by

$$
E_{G}(Q, \hat{P})=-\frac{M}{\pi}-\frac{\pi}{6 M}+\frac{\pi}{M}\left[\frac{Q^{2}}{8}+2 \frac{\hat{P}^{2}}{\pi^{2}}\right]+O\left(M^{-3}\right)
$$

which is the known formula for the ground state in the given charge and momentum sectors. The negative first $1 / M$ term is the familiar zero point energy associated with the closed bosonic string, The ground states in the higher $Q, \hat{P}$ sectors can be easily constructed by applying monomials of suitable eigenoperators to the states of the table, which change to the desired values of $Q, \hat{P}$ creating the lowest possible amount of energy. For example the operator $B_{(M-2) / 4}^{\dagger} B_{(3 M+2) / 4}^{\dagger}$ adds $(-4)$ to $Q$ and 0 to $\hat{P}$ and $2 \pi / M=4^{2} \pi /(8 M)$ to the energy.

\subsection{Energy levels and degeneracies for $M$ large}

Because the system is one of free fermions it is straightforward to read off the energy levels and work out their degeneracies. However, it is useful to organize this information in terms of generating functions, especially when $M$ is large, when the generating functions are elliptic functions. There is a famous identity which codes the known possibility ("bosonization") of describing free fermion fields by free compactified bosonic fields. We quote it in the form ${ }^{4}$

$$
q^{\alpha^{2} / 4} \prod_{n=1}^{\infty}\left(1+e^{2 i \theta} q^{2 n+\alpha-1}\right)\left(1+e^{-2 i \theta} q^{2 n-\alpha-1}\right)=\prod_{n=1}^{\infty}\left(1-q^{2 n}\right)^{-1} \sum_{m=-\infty}^{\infty} q^{(m+\alpha / 2)^{2}} e^{2 i m \theta}
$$

Putting $q=e^{-\pi / M}$ and expansing in powers of $q$ generates the energy spectrum, with the exponents giving the energies and the coefficients the degeneracy of each level. Inspecting the large $M$ energies we see we shall need $\alpha=0,1$ for even $M$, and $\alpha=1 / 2$ for odd $M$. The left side of the identity describes the spectrum in terms of free fermions and the right side as bosonic oscillators.

Let's start with the case where $M \in 4 \mathbb{N}$. The operator $B_{r}^{\dagger}$ is a creation operator for $r \notin G$. It creates low energy for $r$ near $3 M / 4$ or $M / 4$. In the first case we call the operator $b_{r}^{\dagger}$, which creates -2 units of $Q, 1 / 2$ unit of $\hat{P} / \pi$, and an odd multiple of $\pi / M$ in energy. It yields a factor $e^{-2 i \theta+i \phi / 2} q^{2 n-1}$. In the second case we call the operator $\tilde{b}_{r}^{\dagger}$, which creates -2 units of $Q,-1 / 2$ unit of $\hat{P} / \pi$, and an odd multiple of $\pi / M$ in energy. It yields a factor $e^{-2 i \theta-i \phi / 2} q^{2 n-1}$. When $r \in G, B_{r}$ is the creation operator. If $r$ is near $3 M / 4$, it is called

\footnotetext{
${ }^{4}$ In this subsection we follow standard conventions and denote the elliptic function modulus by $q$. suspending the use of $q$ for the number of overturned spins.
} 
$b_{r}$, and creates 2 units of $Q,-1 / 2$ unit of $\hat{P} / \pi$, and an odd multiple of $\pi / M$ in energy. It yields a factor $e^{2 i \theta-i \phi / 2} q^{2 n-1}$. Finally the case $r$ near $M / 4$ yields the factor $e^{2 i \theta+i \phi / 2} q^{2 n-1}$. Thus the generating function in fermion language is

$$
\prod_{n=1}^{\infty}\left(1+e^{-2 i \theta+i \phi / 2} q^{2 n-1}\right)\left(1+e^{-2 i \theta-i \phi / 2} q^{2 n-1}\right)\left(1+e^{2 i \theta-i \phi / 2} q^{2 n-1}\right)\left(1+e^{2 i \theta+i \phi / 2} q^{2 n-1}\right)
$$

Only the terms corresponding to an even number of operators applied to the ground state are relevant, so this formula should be projected onto that sector. This can be done by symmetrizing it under $\theta \rightarrow \theta+\pi / 2$. If we apply the elliptic function identity (with $\alpha=0$ ) twice we arrive at an expression for which the desired projection is transparent. the right side of the identity is

$$
\prod_{n}\left(1-q^{2 n}\right)^{-2} \sum_{k, m} q^{k^{2}+m^{2}} e^{2 i(k+m) \theta+i(k-m) \phi / 2}
$$

In this form the projection is simply to restrict the sum to $k+m$ even. To do this call $k+m=K$, so that $k-m=K-2 m$. If $K$ is even we can define $J=m-K / 2$. Then

$$
m^{2}+k^{2}=(J+K / 2)^{2}+(J-K / 2)^{2}=2 J^{2}+\frac{K^{2}}{2}
$$

and the projected expression is

$$
\prod_{n}\left(1-q^{2 n}\right)^{-2} \sum_{K \operatorname{even}, J} q^{K^{2} / 2+2 J^{2}} e^{2 i K \theta-i J \phi}
$$

From the way $\theta, \phi$ were introduced, it is clear that $J=-\hat{P} / \pi$ and $2 K=Q$, so the exponent of $q$ reads $Q^{2} / 8+2 \hat{P}^{2} / \pi^{2}$, the known result. But this sector only includes charges $Q \in 4 \mathbb{Z}$. the odd multiples of 2 are covered by the $\Omega=-1$ sector built on the second line, which we turn to next.

The state described by the second line of the table has $Q=+2, \hat{P}=0$, and energy $\pi /(2 M)$ above the energy of line 1 . Also the excitation energies are even integers times $\pi / M$. It is then straightforward to establish the generating function:

$$
\begin{aligned}
& e^{2 i \theta} q^{1 / 2}\left(1+e^{-2 i \theta+i \phi / 2}\right)\left(1+e^{-2 i \theta-i \phi / 2}\right) \\
& \prod_{n=1}^{\infty}\left(1+e^{-2 i \theta+i \phi / 2} q^{2 n}\right)\left(1+e^{-2 i \theta-i \phi / 2} q^{2 n}\right)\left(1+e^{2 i \theta-i \phi / 2} q^{2 n}\right)\left(1+e^{2 i \theta+i \phi / 2} q^{2 n}\right) \\
&= q^{1 / 2}\left(e^{i \theta-i \phi / 4}+e^{-i \theta+i \phi / 4}\right)\left(e^{i \theta+i \phi / 4}+e^{-i \theta-i \phi / 4}\right) \\
& \prod_{n=1}^{\infty}\left(1+e^{-2 i \theta+i \phi / 2} q^{2 n}\right)\left(1+e^{-2 i \theta-i \phi / 2} q^{2 n}\right)\left(1+e^{2 i \theta-i \phi / 2} q^{2 n}\right)\left(1+e^{2 i \theta+i \phi / 2} q^{2 n}\right)
\end{aligned}
$$

Of course only the terms corresponding to an even number of excitation above the second line ground state are relevant. These are singled out by antisymmetrizing the whole expression under $\theta \rightarrow \theta+\pi / 2$. Again we can apply the elliptic function (with $\alpha=1$ ) twice to rewrite the generating function

$$
\prod_{n}\left(1-q^{2 n}\right)^{-2} \sum_{k, m} q^{(k+1 / 2)^{2}+(m+1 / 2)^{2}} e^{2 i(k+m+1) \theta+i(k-m) \phi / 2}
$$


The projection is achieved by restricting the $k, m$ sum to $k+m$ even. in terms of the $J, K$ summation indices introduced previously the generating function reads

$$
\prod_{n}\left(1-q^{2 n}\right)^{-2} \sum_{K \text { even }, J} q^{(K+1)^{2} / 2+2 J^{2}} e^{2 i(K+1) \theta-i J \phi}=\prod_{n}\left(1-q^{2 n}\right)^{-2} \sum_{K \text { odd }, J} q^{K^{2} / 2+2 J^{2}} e^{2 i K \theta-i J \phi}
$$

Putting the two sectors together simply relaxes the even or odd restriction:

$$
\prod_{n}\left(1-q^{2 n}\right)^{-2} \sum_{K, J} q^{K^{2} / 2+2 J^{2}} e^{2 i K \theta-i J \phi}, \quad Q \in 2 \mathbb{Z}
$$

The analysis for $M \in 2+4 \mathbb{N}$ is the same in spite of the flip in $\Omega$ assignments because the momenta relative to the Fermi momenta is identical in both cases.

The analysis for odd $M$ is a bit more involved. Starting with the ground state described by line 7 of the table, we read off the generating function:

$$
\begin{aligned}
e^{i \theta-i \phi / 2} q^{5 / 8} & \prod_{n=0}^{\infty}\left(1+e^{-2 i \theta+i \phi / 2} q^{2 n-1 / 2}\right)\left(1+e^{-2 i \theta-i \phi / 2} q^{2 n+3 / 2}\right)\left(1+e^{2 i \theta-i \phi / 2} q^{2 n+5 / 2}\right)\left(1+e^{2 i \theta+i \phi / 2} q^{2 n+1 / 2}\right) \\
= & e^{i \theta-i \phi / 2} q^{5 / 8} \prod_{n=1}^{\infty}\left(1+e^{2 i \theta+i \phi / 2} q^{2 n-3 / 2}\right)\left(1+e^{-2 i \theta-i \phi / 2} q^{2 n=1 / 2}\right) \\
& \prod_{n=1}^{\infty}\left(1+e^{-2 i \theta+i \phi / 2} q^{2 n-5 / 2}\right)\left(1+e^{2 i \theta-i \phi / 2} q^{2 n+1 / 2}\right)
\end{aligned}
$$

We use the elliptic function identities for $\alpha=-1 / 2$ and $\alpha=3 / 2$ to arrive at the bosonic form

$$
\begin{aligned}
e^{i \theta-i \phi / 2} \prod_{n}\left(1-q^{2 n}\right)^{-2} \sum_{k, m} q^{(k-1 / 4)^{2}+(m+3 / 4)^{2}} e^{2 i(k+m+1) \theta+i(k-m) \phi / 2} \\
=\prod_{n}\left(1-q^{2 n}\right)^{-2} \sum_{k, m} q^{(k-1 / 4)^{2}+(m+3 / 4)^{2}} e^{i(2(k+m)+1) \theta+i(k-m-1) \phi / 2}
\end{aligned}
$$

Again only the terms with $k+m$ even are relevant. With this restriction the change of indices $k=K / 2-J, m=K / 2+J$ is valid, so

$$
\begin{aligned}
(k-1 / 4)^{2}+(m+3 / 4)^{2} & =(K / 2-J-1 / 4)^{2}+(K / 2+J+3 / 4)^{2} \\
& =2(K / 2+1 / 4)^{2}+2(J+1 / 2)^{2}
\end{aligned}
$$

yielding the result

$$
\prod_{n}\left(1-q^{2 n}\right)^{-2} \sum_{K \operatorname{even}, J} q^{(2 K+1)^{2} / 8+2(J+1 / 2)^{2}} e^{i(2 K+1) \theta-i(J+1 / 2) \phi}
$$


Considering the states built on line 9 of the table, shows that the generating function for such excited states is the complex conjugate of the one just computed for line 7 :

$$
\begin{aligned}
\prod_{n} & \left(1-q^{2 n}\right)^{-2} \sum_{K \text { even }, J} q^{(2 K+1)^{2} / 8+2(J+1 / 2)^{2}} e^{-i(2 K+1) \theta+i(J+1 / 2) \phi} \\
& =\prod_{n}\left(1-q^{2 n}\right)^{-2} \sum_{K \text { even }, J} q^{(2 K-1)^{2} / 8+2(J-1 / 2)^{2}} e^{i(2 K-1) \theta-i(J-1 / 2) \phi} \\
& =\prod_{n}\left(1-q^{2 n}\right)^{-2} \sum_{K \operatorname{even}, J} q^{(2(K-1)+1)^{2} / 8+2(J+1 / 2)^{2}} e^{i(2(K-1)+1) \theta-i(J+1 / 2) \phi} \\
& =\prod_{n}\left(1-q^{2 n}\right)^{-2} \sum_{K \text { odd }, J} q^{(2 K+1)^{2} / 8+2(J+1 / 2)^{2}} e^{i(2 K+1) \theta-i(J+1 / 2) \phi}
\end{aligned}
$$

In successive lines we have reversed the signs of $K, J$, Shifted $J$ by one unit, and shifted $K$ by one unit making it odd rather than even, Thus putting the two sectors together simply relaxes the restrictions on the range of $K$,

$$
\prod_{n}\left(1-q^{2 n}\right)^{-2} \sum_{K, J} q^{(2 K+1)^{2} / 8+2(J+1 / 2)^{2}} e^{i(2 K+1) \theta-i(J+1 / 2) \phi}, \quad Q \in 1+2 \mathbb{Z}
$$

In spite of the need to allow for 8 base ground states, only four lead to distinct outcomes for energies and degeneracies. They can be characterized by the nature of $Q$ :

$$
Q \in 4 \mathbb{Z}, \quad Q \in 2+4 \mathbb{Z}, \quad Q \in 1+4 \mathbb{Z}, \quad Q \in-1+4 \mathbb{Z}
$$

\section{Conformal invariance and bosonization at large $M$}

\subsection{Virasoro generators}

We can summarize the spectrum using energy eigenoperators by writing

$$
\begin{aligned}
H & =\sum_{r} \cos \frac{2 \pi r}{M} B_{r}^{\dagger} B_{r} \\
& =E_{G}^{\Omega}+\sum_{r \notin G} \cos \frac{2 \pi r}{M} B_{r}^{\dagger} B_{r}-\sum_{r \in G} \cos \frac{2 \pi r}{M} B_{r} B_{r}^{\dagger}
\end{aligned}
$$

And we remember that $r \in 1 / 2+\mathbb{Z}$ when $\Omega=+1$, and $r \in \mathbb{Z}$ when $\Omega=-1$. When $M$ is large $B_{r}^{\dagger}$ creates energies of order $1 / M$ for $r$ near $3 M / 4$ or near $M / 4$. In the first case we call the operator $b_{r-3 M / 4}^{\dagger}$ and in the second case $\tilde{b}_{r-M / 4}^{\dagger}$. Then we can write the effective low energy Hamiltonian

$$
H_{\mathrm{eff}}=E_{G}^{\Omega}+\frac{2 \pi}{M}\left[\sum_{s \notin G} s b_{s}^{\dagger} b_{s}+\sum_{t \notin G} t \tilde{b}_{t}^{\dagger} \tilde{b}_{t}+\sum_{u \in G}(-u) b_{u} b_{u}^{\dagger}+\sum_{v \in G}(-v) \tilde{b}_{v} \tilde{b}_{v}^{\dagger}\right]
$$


We have written each summation index as a different letter, because their ranges can be different depending on the nature of $Q$ :

$$
\begin{array}{llll}
Q \in 4 \mathbb{Z}: & s, t, u, v \in \frac{1}{2}+\mathbb{Z}, & s, t>0, & u, v<0 \\
Q \in 2+4 \mathbb{Z}: & s, t, u, v \in \mathbb{Z}, & s, t \geq 0, & u, v<0 \\
Q \in 1+4 \mathbb{Z}: & s, t, u, v \in-\frac{1}{4}+\mathbb{Z}, & s \geq-\frac{1}{4}, & t \geq \frac{3}{4}, \quad u \leq-\frac{5}{4}, \quad v \leq-\frac{1}{4} \\
Q \in-1+4 \mathbb{Z}: & s, t, u, v \in \frac{1}{4}+\mathbb{Z}, & s \geq \frac{5}{4}, & t \geq \frac{1}{4}, \quad u \leq \frac{1}{4}, \quad v \leq-\frac{3}{4}
\end{array}
$$

We list explicitly the Hamiltonian for each case, dropping the term in $E_{G}$ linear in $M$ :

$$
\begin{aligned}
H_{\text {eff }}= & -\frac{\pi}{6 M}+\frac{2 \pi}{M} \sum_{n=0}^{\infty}\left[\left(n+\frac{1}{2}\right) b_{n+1 / 2}^{\dagger} b_{n+1 / 2}+\left(n+\frac{1}{2}\right) \tilde{b}_{n+1 / 2}^{\dagger} \tilde{b}_{n+1 / 2}\right] \\
& +\frac{2 \pi}{M} \sum_{n=0}^{\infty}\left[\left(n+\frac{1}{2}\right) b_{-n-1 / 2} b_{-n-1 / 2}^{\dagger}+\left(n+\frac{1}{2}\right) \tilde{b}_{-n-1 / 2} \tilde{b}_{-n-1 / 2}^{\dagger}\right], \quad Q \in 4 \mathbb{Z} \\
H_{\text {eff }}= & -\frac{\pi}{6 M}+\frac{\pi}{2 M}+\frac{2 \pi}{M} \sum_{n=1}^{\infty} n\left[b_{n}^{\dagger} b_{n}+\tilde{b}_{n}^{\dagger} \tilde{b}_{n}+b_{-n} b_{-n}^{\dagger}+\tilde{b}_{-n} \tilde{b}_{-n}^{\dagger}\right], \quad Q \in 2+4 \mathbb{Z} \\
H_{\text {eff }}= & -\frac{\pi}{6 M}+\frac{5 \pi}{8 M}+\frac{2 \pi}{M} \sum_{n=0}^{\infty}\left[\left(n-\frac{1}{4}\right) b_{n-1 / 4}^{\dagger} b_{n-1 / 4}+\left(n+\frac{3}{4}\right) \tilde{b}_{n+3 / 4}^{\dagger} \tilde{b}_{n+3 / 4}\right] \\
& +\frac{2 \pi}{M} \sum_{n=0}^{\infty}\left[\left(n+\frac{5}{4}\right) b_{-n-5 / 4} b_{-n-5 / 4}^{\dagger}+\left(n+\frac{1}{4}\right) \tilde{b}_{-n-1 / 4} \tilde{b}_{-n-1 / 4}^{\dagger}\right], \quad Q \in 1+4 \mathbb{Z} \\
H_{\text {eff }}= & -\frac{\pi}{6 M}+\frac{5 \pi}{8 M}+\frac{2 \pi}{M} \sum_{n=0}^{\infty}\left[\left(n+\frac{5}{4}\right) b_{n+5 / 4}^{\dagger} b_{n+5 / 4}+\left(n+\frac{1}{4}\right) \tilde{b}_{n+1 / 4}^{\dagger} \tilde{b}_{n+1 / 4}\right] \\
& +\frac{2 \pi}{M} \sum_{n=0}^{\infty}\left[\left(n-\frac{1}{4}\right) b_{-n+1 / 4} b_{-n+1 / 4}^{\dagger}+\left(n+\frac{3}{4}\right) \tilde{b}_{-n-3 / 4} \tilde{b}_{-n-3 / 4}^{\dagger}\right], \quad Q \in-1+4 \mathbb{Z}
\end{aligned}
$$

The operator products in the above expressions are all normal ordered relative to the ground state in each sector, that is operators which annihilate the ground state are on the right. If we understand the double colon notation to mean normal ordering in this sense, we can give these expressions more compact forms:

$$
\begin{aligned}
& H_{\mathrm{eff}}^{0}=-\frac{\pi}{6 M}+\frac{2 \pi}{M} \sum_{n=-\infty}^{\infty}\left(n+\frac{1}{2}\right)\left[: b_{n+1 / 2}^{\dagger} b_{n+1 / 2}:+: \tilde{b}_{n+1 / 2}^{\dagger} \tilde{b}_{n+1 / 2}:\right] \\
& H_{\mathrm{eff}}^{2}=-\frac{\pi}{6 M}+\frac{\pi}{2 M}+\frac{2 \pi}{M} \sum_{n=-\infty}^{\infty} n\left[: b_{n}^{\dagger} b_{n}:+: \tilde{b}_{n}^{\dagger} \tilde{b}_{n}:\right] \\
& H_{\mathrm{eff}}^{1}=-\frac{\pi}{6 M}+\frac{5 \pi}{8 M}+\frac{2 \pi}{M} \sum_{n=-\infty}^{\infty}\left(n-\frac{1}{4}\right)\left[: b_{n-1 / 4}^{\dagger} b_{n-1 / 4}:+: \tilde{b}_{n-1 / 4}^{\dagger} \tilde{b}_{n-1 / 4}:\right] \\
& H_{\mathrm{eff}}^{-1}=-\frac{\pi}{6 M}+\frac{5 \pi}{8 M}+\frac{2 \pi}{M} \sum_{n=-\infty}^{\infty}\left(n+\frac{1}{4}\right)\left[: b_{n+1 / 4}^{\dagger} b_{n+1 / 4}:+: \tilde{b}_{n+1 / 4}^{\dagger} \tilde{b}_{n+1 / 4}:\right]
\end{aligned}
$$

In each sector, the Hamiltonian is a sum of two commuting operators, one depending only on $b$ and the other on $\tilde{b}$. Each can be identified with the zero member of commuting 
Virasoro generators, $L_{n}, \tilde{L}_{n}$. The Virasoro algebras must take the form

$$
\left[L_{n}, L_{m}\right]=(n-m) L_{n+m}+C_{n} \delta_{n,-m}
$$

and similarly for $\tilde{L}_{n}$. When $n \neq 0$, there is no ordering ambiguity in the definition of $L_{n}$ so allowing for fractional modes we may write

$$
L_{n} \equiv \sum_{k=-\infty}^{\infty}(k+n / 2+\alpha) b_{k+\alpha}^{\dagger} b_{k+n+\alpha}, \quad n \neq 0
$$

This expression is ambiguous up to an additive constant for $n=0$. But $L_{0}$ can be obtained using the algebra of the unambiguous $L_{n}$ : namely $\left[L_{n}, L_{-n}\right]=2 n L_{0}+C_{n}$, once conventions are set in determining $C_{n}$. Those conventions will be set by identifying the $L_{0}, \tilde{L}_{0}$ with pieces of the Hamiltonians in the various sectors. Inspection shows that the appropriate values of $\alpha$ are $1 / 2,0,1 / 4,-1 / 4$ for the respective sectors.

It is easy to confirm the Virasoro algebra for $n \neq-m$, but the determination of the $C_{n}$ is more involved.

$$
\begin{aligned}
L_{0}^{0} & =\sum_{n=0}^{\infty}\left(n+\frac{1}{2}\right)\left[b_{n+1 / 2}^{\dagger} b_{n+1 / 2}+b_{-n-1 / 2} b_{-n-1 / 2}^{\dagger}\right] \\
\tilde{L}_{0}^{0} & =\sum_{n=0}^{\infty}\left(n+\frac{1}{2}\right)\left[\tilde{b}_{n+1 / 2}^{\dagger} \tilde{b}_{n+1 / 2}+\tilde{b}_{-n-1 / 2} \tilde{b}_{-n-1 / 2}^{\dagger}\right] \\
L_{0}^{2} & =\sum_{n=0}^{\infty}(n)\left[b_{n}^{\dagger} b_{n}+b_{-n} b_{-n}^{\dagger}\right], \quad \tilde{L}_{0}^{0}=\sum_{n=0}^{\infty}(n)\left[\tilde{b}_{n}^{\dagger} \tilde{b}_{n}+\tilde{b}_{-n} \tilde{b}_{-n}^{\dagger}\right] \\
L_{0}^{1} & =\sum_{n=0}^{\infty}\left[\left(n-\frac{1}{4}\right) b_{n-1 / 4}^{\dagger} b_{n-1 / 4}+\left(n+\frac{5}{4}\right) b_{-n-5 / 4} b_{-n-5 / 4}^{\dagger}\right] \\
\tilde{L}_{0}^{1} & =\sum_{n=0}^{\infty}\left[\left(n+\frac{3}{4}\right) \tilde{b}_{n+3 / 4}^{\dagger} \tilde{b}_{n+3 / 4}+\left(n+\frac{1}{4}\right) \tilde{b}_{-n-1 / 4} \tilde{b}_{-n-1 / 4}^{\dagger}\right] \\
L_{0}^{-1} & =\sum_{n=0}^{\infty}\left[\left(n+\frac{5}{4}\right) b_{n+5 / 4}^{\dagger} b_{n+5 / 4}+\left(n-\frac{1}{4}\right) b_{-n+1 / 4} b_{-n+1 / 4}^{\dagger}\right] \\
\tilde{L}_{0}^{-1} & =\sum_{n=0}^{\infty}\left[\left(n+\frac{1}{4}\right) \tilde{b}_{n+1 / 4}^{\dagger} \tilde{b}_{n+1 / 4}+\left(n+\frac{3}{4}\right) \tilde{b}_{-n-3 / 4} \tilde{b}_{-n-3 / 4}^{\dagger}\right]
\end{aligned}
$$

For each of the four cases, we have 2 commuting Virasoro algebras, with potentially different central terms for each. With the above identifications for $L_{0}$ in each case, careful calculation shows

$$
\begin{array}{rlrl}
C_{n}^{0} & =\tilde{C}_{n}^{0}=\frac{1}{12}\left(n^{3}-n\right) & & \\
C_{n}^{2} & =\tilde{C}_{n}^{2}=\frac{1}{12}\left(n^{3}+2 n\right) & & \\
C_{n}^{1} & =\frac{1}{12}\left(n^{3}+3 n / 4\right), & \tilde{C}_{n}^{1}=\frac{1}{12}\left(n^{3}-n / 4\right) \\
C_{n}^{-1} & =\frac{1}{12}\left(n^{3}+3 n / 4\right), & \tilde{C}_{n}^{-1} & =\frac{1}{12}\left(n^{3}-n / 4\right)
\end{array}
$$


The coefficient of $n^{3}$ is universal corresponding to a single bosonic worldsheet coordinate. This term is controlled by short distances and is expected to be independent of the structure of the ground state. In contrast, the linear term varies from one case to another. It is perhaps more natural to include the linear term in the definition of $L_{0}$, keeping only the cubic term in the definition of $C_{n}$. If this is done one finds that the constant terms in the Hamiltonian are precisely accounted for with the simple identification $H=2 \pi\left(L_{0}+\tilde{L}_{0}\right) / M$.

The asymmetry between $C$ and $\tilde{C}$ in the cases that $Q$ is odd, though a little startling, is understandable given that the occupied states in the chosen vacuum are asymmetric. As seen in the table the ground energy level with $Q=1$ is doubly degenerate, and choosing the other ground state reverses the asymmetry.

\subsection{Bosonization of the spin system at $\Delta=0$}

The equivalence of free fermion systems to bosonic systems in one space dimension is very well studied. In this subsection we quote the equivalence in the operator language for the Heisenberg spin system studied in this article. There are four sectors, each of which is described by two anticommuting sets of fermion operators $b, \tilde{b}$, so all together eight slightly different bosonization formulas. Each set is characterized by a fractional mode number, which we have called $\alpha$. We first quote the formulas for general $\alpha$, and use $b$ for the Fermi description, and $a$ for the bosonic description.

We write the boson as a bilinear of fermions

$$
a_{n}=\sum_{k=-\infty}^{\infty}: b_{k+\alpha}^{\dagger} b_{k+n+\alpha}:
$$

where we fix the normal ordering with respect to a vacuum satisfying $b_{k+\alpha}|0\rangle=0$ for $k \geq 0$ and $b_{k+\alpha}^{\dagger}|0\rangle=0$ for $k<0$. it follows from this formula and the anticommutation relations $\left\{b_{k+\alpha}, b_{l+\alpha}^{\dagger}\right\}=\delta_{k l}$ that

$$
\left[a_{n}, a_{m}\right]=n \delta_{n,-m}, \quad a_{n}^{\dagger}=a_{-n}, \quad a_{n}|0\rangle=0, \quad n \geq 0 .
$$

When calculating the commutator care must be taken to respect normal ordering: each term in the commutator should be separately normal ordered via Wick;s theorem, after which the operator parts cancel leaving behind the right side of the equation.

Bosonization in one space dimension, remarkably, leads to an equality between two relatively simple Hamiltonians expressed in terms of bosonic or fermionic degrees of freedom. For this system at the free fermion point this relation holds for the entire Virasoro algebra. The fermion form of the Virasoro algebra was given in the previous subsection. The bosonic form is given $b y^{5}$

$$
L_{n}=\beta a_{n}+\frac{1}{2} \sum_{m=-\infty}^{\infty}: a_{-m} a_{m+n}:
$$

\footnotetext{
${ }^{5}$ More generally one could also add a term $i n \gamma a_{n}$ to $L_{n}$ which modifies the cubic term in $C_{n}$, but since the two systems already agree on the cubic term, $\gamma=0$ for us.
} 
Here normal ordering simply means positive moded $a$ 's always stand to the right of negative moded $a$ 's. Since we will be inserting the bosonization formula, which uses its own normal ordering prescription, we write out $L_{n}$ explicitly:

$$
\begin{aligned}
L_{n} & =\beta a_{n}+\frac{1}{2} \sum_{m=0}^{\infty} a_{-m} a_{m+n}+\frac{1}{2} \sum_{m=-\infty}^{-1} a_{m+n} a_{-m} \\
& =\beta a_{n}+\frac{1}{2} \sum_{m=0}^{\infty} a_{-m} a_{m+n}+\frac{1}{2} \sum_{m=1}^{\infty} a_{-m+n} a_{m}
\end{aligned}
$$

so that normal ordering is no longer needed.

Now one can plug the bosonization formula into $L_{n}(a)$ and, with due attention to operator ordering, obtain

$$
L_{n}(a)=\sum_{k=-\infty}^{\infty}(k+n / 2+\beta+1 / 2): b_{k+\alpha}^{\dagger} b_{k+n+\alpha}:
$$

Comparison gives $\beta=\alpha-1 / 2$.

In bosonic language the $\beta=\alpha-1 / 2$ term in $L_{n}$ is equivalent to the replacement $a_{0} \rightarrow a_{0}+\beta$ for $n \neq 0$. For $n=0$ the $a_{0}$ dependence is

$$
\frac{a_{0}^{2}}{2}+\beta a_{0}=\frac{\left(a_{0}+\beta\right)^{2}}{2}-\frac{\beta^{2}}{2}
$$

so in addition to the substitution $a_{0} \rightarrow a_{0}+\beta$ a constant term added to $L_{0}$ appears. With this definition of $L_{0}$ the c-number term in the Virasoro algebra assumes the form $C_{n}=\beta^{2}+\left(n^{3}-n\right) / 12$. Clearly if one removes that extra term from $L_{0}$ and puts it in the c-number term, the latter becomes $C_{n} \rightarrow\left(n^{3}-n\right) / 12$, the value it has at $\beta=0$. In short, with the new definitions

$$
\begin{aligned}
a_{0} & =\sum_{k=-\infty}^{\infty}: b_{k+\alpha}^{\dagger} b_{k+\alpha}+\alpha-\frac{1}{2}: \\
L_{n} & =\sum_{k=-\infty}^{\infty}(k+n / 2+\alpha): b_{k+\alpha}^{\dagger} b_{k+n+\alpha}:+\frac{1}{2}\left(\alpha-\frac{1}{2}\right)^{2} \delta_{n 0},
\end{aligned}
$$

the $L_{n}$, whose bosonic form is $L_{n}=\sum_{m}: a_{-m} a_{m+n}: / 2$, satisfy the standard Virasoro algebra for transverse dimension $d=1$

$$
\left[L_{n}, L_{m}\right]=(n-m) L_{n+m}+\frac{1}{12}\left(n^{3}-n\right) \delta_{n,-m}
$$




\subsection{Interpretation of zero mode operators}

The zero modes $a_{0}, \tilde{a}_{0}$ can be linked to the two conserved operators $Q$ and $\hat{P} / \pi$, whose values for the ground states are listed in the table:

$$
\begin{aligned}
Q^{0} & =-2 \sum_{n=0}^{\infty}\left[: b_{n+1 / 2}^{\dagger} b_{n+1 / 2}:+: \tilde{b}_{n+1 / 2}^{\dagger} \tilde{b}_{n+1 / 2}:\right]=-2\left(a_{0}+\tilde{a}_{0}\right) \\
\frac{\hat{P}^{0}}{\pi} & =\frac{!}{2} \sum_{n=0}^{\infty}\left[: b_{n+1 / 2}^{\dagger} b_{n+1 / 2}:-{ }^{`}: \tilde{b}_{n+1 / 2}^{\dagger} \tilde{b}_{n+1 / 2}:\right]=\frac{1}{2}\left(a_{0}-\tilde{a}_{0}\right) \\
Q^{2} & =2-2 \sum_{n=0}^{\infty}\left[: b_{n}^{\dagger} b_{n}:+: \tilde{b}_{n}^{\dagger} \tilde{b}_{n}:\right]=-2\left(a_{0}+\tilde{a}_{0}\right) \\
\frac{\hat{P}^{2}}{\pi} & =\frac{!}{2} \sum_{n=0}^{\infty}\left[: b_{n}^{\dagger} b_{n}:-: \tilde{b}_{n}^{\dagger} \tilde{b}_{n}:\right]=\frac{1}{2}\left(a_{0}-\tilde{a}_{0}\right) \\
Q^{1} & =1-2 \sum_{n=0}^{\infty}\left[: b_{n-1 / 4}^{\dagger} b_{n-1 / 4}:+: \tilde{b}_{n+3 / 4}^{\dagger} \tilde{b}_{n+3 / 4}:\right]=-2\left(a_{0}+\tilde{a}_{0}\right) \\
\frac{\hat{P}^{1}}{\pi} & =-\frac{!}{2}+\frac{!}{2} \sum_{n=0}^{\infty}\left[: b_{n+3 / 4}^{\dagger} b_{n+3 / 4}:-: \tilde{b}_{n+3 / 4}^{\dagger} \tilde{b}_{n+3 / 4}:\right]=\frac{1}{2}\left(a_{0}-\tilde{a}_{0}\right) \\
Q^{-1} & =-1-2 \sum_{n=0}^{\infty}\left[: b_{n+5 / 4}^{\dagger} b_{n+5 / 4}:+: \tilde{b}_{n+1 / 4}^{\dagger} \tilde{b}_{n+1 / 4}:\right]=-2\left(a_{0}+\tilde{a}_{0}\right) \\
\frac{\hat{P}^{-1}}{\pi} & =\frac{!}{2}+\frac{!}{2} \sum_{n=0}^{\infty}\left[: b_{n+5 / 4}^{\dagger} b_{n+5 / 4}:-: \tilde{b}_{n+1 / 4}^{\dagger} \tilde{b}_{n+1 / 4}:\right]=\frac{1}{2}\left(a_{0}-\tilde{a}_{0}\right)
\end{aligned}
$$

in all cases the new definitions of $a_{0}$ and $\tilde{a}_{0}$ are used on the extreme right side. In a similar vein, using the new definition of $L_{0}, \tilde{L}_{0}$, the Hamiltonian is given by

$$
H=-\frac{\pi}{6 M}+\frac{2 \pi}{M}\left(L_{0}+\tilde{L}_{0}\right)
$$

Furthermore if $L_{0}, \tilde{L}_{0}$ are defined so that the c-number term in the Virasoro algebra is purely cubic in $n$, the $-\pi /(6 M)$ term on the right of the above equation should be deleted.

\section{Spin chain contribution to 3 string vertex}

\subsection{Preliminary discussion}

Perturbative string interactions are efficiently obtained using worldsheet path integrals. In lightcone parameters, using Mandelstam's interacting string formalism, any worldsheet can be tessellated by a series of rectangular pieces each describing the propagation of a free string. All interactions can be built up from the basic three string vertex at which a long string whose propagator is a rectangle of width $P_{1}^{+}+P_{2}^{+}$makes an instantaneous transition to or from two shorter strings whose propagators have width $P_{1}^{+}$and $P_{2}^{+}$. Factorizing each propagator shows that the vertex is nothing more than the overlap of the long string wave function with the product of the two short string wave functions, the amplitude for the transition in one direction being the complex conjugate of the amplitude for the time reversed transition.

A given string theory is defined by a number of worldsheet fields, typically including bosonic coordinates compactified or not, Grassmann fields representing spin degrees of 
freedom, or more generally any worldsheet system that supports the Virasoro algebra. In our string bit models the worldsheet is not fundamental, but rather is a composite structure describing the evolution of chains of string bits with time. Any fields living on the worldsheet must arise from internal degrees of freedom carried by the string bit, and indeed need arise from only a finite number of bit degrees of freedom. In this paper, we are exploring the possibility that each spin of the Heisenberg system resides on a single string bit. Then the Heisenberg chain arises when a large number of bits link together. To the extent that the effective worldsheet fields arising in this way are decoupled from each other the basic overlap is just a product of overlaps for each subsystem. Then we can focus on the overlap of the Heisenberg wave functions alone, as we have done in previous sections.

Away from the free fermion point $(\Delta \neq 0)$ the Hamiltonian has terms up to quartic in fermion fields, making the overlap calculation very difficult. However, by bosonizing the system, we obtain a Hamiltonian quadratic in boson fields, with zero modes quantized according to the eigenvalues of $Q$ and $\hat{P} / \pi$. We interpret $Q$ as a Kaluza-Klein momentum of a compactified coordinate and $\hat{P} / \pi$ as the associated winding number. Our strategy for obtaining the overlap even with $\Delta \neq 0$ is to use Mandelstam's interacting string formalism for bosonic string, generalized to allow for compactified coordinates. The only loose end in this approach, is figuring out how to deal with the two zero modes. If all three states participating in the overlap have $\hat{P}=0$, it is probably safe to use the Mandelstam vertex for continuous (uncompactified) momenta by simply setting those momenta equal to the desired KK momentum proportional to $Q$. This is because $Q=Q_{1}+Q_{2}$ is an operator equality, showing that the vertex conserves $Q$. The same is not true of the momentum operator, so the possibility that the vertex violates $\hat{P}$ conservation can not be ruled out. Indeed, this is known to occur for the Green-Schwarz worldsheet spinors. We shall find in the next subsection that for the Heisenberg spin system $\hat{P}$ is indeed conserved in the string limit.

\section{$5.2 \quad$ Three string overlap}

In string theory the interactions of strings arise from instantaneous changes in the Hamiltonian, corresponding to the transition of a single closed string to two smaller closed strings. In the string bit approach this process does not change the number of string bits. The overlap involves all available degrees of freedom, but here we only deal with those described by Heisenberg spins. The Hamiltonian for any single spin chain is the one just described. Let's say that the initial chain has $M$ spins, $S_{1}, \ldots, S_{M}$ and the smaller chains have $K$ and $L=M-K$ spins respectively. We identify $S_{1}, \ldots, S_{K}$ as the spin variables of the first small chain and $S_{K+1}, \ldots, S_{M}$ as those of the second small chain,

We construct the raising and lowering operators for the three participating chains

$$
\begin{aligned}
B_{r} & =\frac{1}{\sqrt{M}} \sum_{k=1}^{M} e^{-2 \pi i k r / M} S_{k}, & & 0 \leq r<M \\
b_{r}^{1} & =\frac{1}{\sqrt{K}} \sum_{k=1}^{K} e^{-2 \pi i k r / K} S_{k}, & & \leq r<K \\
b_{r}^{2} & =\frac{1}{\sqrt{L}} \sum_{k=K+1}^{M} e^{-2 \pi i(k-K) r / L} S_{k}, & & 0 \leq r<L
\end{aligned}
$$


To apply the overlap construction developed in section 2, we need the matrix $D=\left(D^{1} \mid D^{2}\right)$

$$
\begin{aligned}
D_{s r}^{1} & =\left\{b_{r}^{1}, B_{s}^{\dagger}\right\}=\frac{1}{\sqrt{K M}} \sum_{k=1}^{K} e^{2 \pi i k(s / M-r / K)} \\
& = \begin{cases}\sqrt{\frac{K}{M}}, & (s / M-r / K) \in \mathbb{Z} \\
-\frac{1}{\sqrt{K M}} \frac{1-e^{2 \pi i(s K / M-r)}}{1-e^{-2 \pi i(s / M-r / K)}}, & (s / M-r / K) \notin \mathbb{Z}\end{cases} \\
D_{s r}^{2} & =\left\{b_{r}^{2}, B_{s}^{\dagger}\right\}=\frac{1}{\sqrt{L M}} \sum_{k=K+1}^{M} e^{2 \pi i(k s / M-(k-K) r / L)}=\frac{1}{\sqrt{L M}} e^{2 \pi i K s / M} \sum_{k=1}^{L} e^{2 \pi i(k s / M-k r / L)}
\end{aligned}
$$

The special cases can easily be seen to follow from a limit of the generic cases, assuming $K, L, M$ are continuous variables. It is only necessary to treat them separately in evaluations (e.g. on a computer) which explicitly require $K, L, M$ to be integers.

We also need a common energy eigenstate, which we called $|0\rangle$ in section 2. A convenient choice is the state in which all spins are up, $\sigma_{k}^{z}|0\rangle=|0\rangle$. This state is an eigenstate of $\Omega$ with value +1 and satisfies $S_{k}|0\rangle=0, B_{k}|0\rangle=0, b_{k}^{1}|0\rangle=0$, and $b_{k}^{2}|0\rangle=0$, for all $k$. It is also an eigenstate of the Hamiltonian, even for $\Delta \neq 0$

$$
H_{\Delta}|0\rangle=\Delta \sum_{k} \sigma_{k}^{z} \sigma_{k+1}^{z}|0\rangle=M \Delta|0\rangle
$$

We shall refer to $|0\rangle$ as the "empty" state. It is not the ground state of either system because there are negative energy levels in both.

Energy eigenstates can be constructed by applying monomials of the $B^{\dagger}$ 's or monomials of $b^{1 \dagger}, b^{2 \dagger}$ 's to the empty state, In the first case one builds energy eigenstates of $H_{1}$ and in the second case those of $H_{2}$, Even monomials give eigenstates with $\Omega=+1$ and odd monomials give eigenstates with $\Omega=-1$. In the case of $H_{1}$ which describes a single spin chain, the operators that go into the even monomials carry half odd integer indices and those going into the odd monomials integer indices. In the case of $H_{2}$, which describes two spin chains with independent operators for each, the operators that go into the even monomials either have half odd integer indices for both chains or integer indices for both chains, and those going into the odd monomials have half odd integer indices for either of the two chains but integer indices for the other one.

In order to make efficient use of the coherent state formalism, we consider two distinct Hamiltonians for each chain: $H^{+}$for which $\Omega$ has been set equal to +1 and $H^{-}$for which $\Omega$ has been set equal to -1 . Eigenstates of $H^{ \pm}$with $\Omega=\mp 1$ are not eigenstates of $H$ and must be discarded. But eigenstates of $H^{ \pm}$with $\Omega= \pm 1$ are eigenstates of $H$, and together span the eigenspace. For the three chain vertex, one does four separate calculations for the Hamiltonian choices $+\rightarrow++,+\rightarrow--,-\rightarrow+-$, and $-\rightarrow-+$. At the end of the calculations, one discards the monomial choices that violate the required correlation between $\Omega$ value and index. 
In section 2 we gave the generic construction of the vertex for systems of free fermions summarized in (2.14). We need to form four (not necessarily square) matrices from $D_{s r}$, where $s$ labels the modes of the long chain, and $r$ labels the modes of the two short strings:

$$
D_{s r}^{G g}, \quad D_{s r}^{\tilde{G} g}, \quad D_{s r}^{G \tilde{g}}, \quad D_{s r}^{\tilde{G} \tilde{g}} .
$$

Here the superscript $G$ means $s$ labels the levels filled in $G, \tilde{G}$ means $s$ labels the levels that are empty in $G$, with a similar meaning for the superscripts $g, \tilde{g}$ with respect to the $r$ index. Since the overlap construction requires the inverse of the first of these 4 matrices, it is important that $D^{G g}$ be a square matrix. ${ }^{6}$ Inspection of our table 1 in all of the numerous allowed cases confirms this to be true. To illustrate consider the cases in which the bit numbers of all three strings are multiples of 4 , so that we can limit attention to the first three lines of the table. The symbol $q$ is the number of levels filled in the corresponding ground state. Thus there are $M / 2$ (or $K / 2$ or $L / 2$ ) labels for the state described by the first line, $(M \mp 2) / 2$ (or $(K \mp 2 / 2$ or $(L \mp 2) / 2)$ for the state described by the second and third line respectively. One immediately sees that a square matrix $q=q_{1}+q_{2}$ is equivalent to charge conservation $Q=Q_{1}+Q_{2}$. Here we are presuming that $\langle G \mid g\rangle \neq 0$.

The 3 chain overlap becomes the three string vertex in the limit that each chain has an infinite number of bits: $M, K, L=M-K \rightarrow \infty$ at fixed ratio $0<x=K / M<1$. In this limit the relevant modes on each string are those with either $r-M_{k} / 4$ of $r-3 M_{k} / 4$ fixed. To implement the simplifications of this stringy limit on the overlap matrices $D^{1}, D^{2}$ we have to consider four cases for each. Define

$$
\begin{aligned}
s^{\prime} & \equiv s-\frac{3 M}{4}, & \tilde{s}^{\prime} & \equiv s-\frac{M}{4} \\
r^{\prime} & \equiv r-\frac{3 K}{4}, & \tilde{r}^{\prime} & \equiv r-\frac{K}{4} \\
t^{\prime} & \equiv t-\frac{3 L}{4}, & \tilde{t}^{\prime} & \equiv t-\frac{L}{4}
\end{aligned}
$$

which are held fixed as $M \rightarrow \infty$ Then in the string limit

$$
\begin{aligned}
D_{s r}^{1} \approx & \begin{cases}-\sqrt{x} \frac{1-\exp \left\{2 \pi i\left(\tilde{s}^{\prime} x-\tilde{r}^{\prime}\right)\right\}}{2 \pi i\left(\tilde{s}^{\prime} x-\tilde{r}^{\prime}\right)}, & \tilde{s}^{\prime}, \tilde{r}^{\prime} \text { fixed } \\
-\sqrt{x} \frac{1-\exp \left\{2 \pi i\left(s^{\prime} x-r^{\prime}\right)\right\}}{2 \pi i\left(s^{\prime} x-r^{\prime}\right)}, & s^{\prime}, r^{\prime} \text { fixed } \\
O\left(\frac{1}{M}\right) & \tilde{s}^{\prime}, r^{\prime} \text { fixed } \\
O\left(\frac{1}{M}\right) & s^{\prime}, \tilde{r}^{\prime} \text { fixed }\end{cases} \\
D_{s r}^{2} \approx & \begin{cases}-i^{K} \sqrt{1-x} \frac{\exp \left\{2 \pi i \tilde{s}^{\prime} x\right\}-\exp \left\{2 \pi i\left(\tilde{s}^{\prime}-\tilde{t}^{\prime}\right)\right\}}{2 \pi i\left(\tilde{s}^{\prime}(1-x)-\tilde{t}^{\prime}\right)}, & \tilde{s}^{\prime}, \tilde{t}^{\prime} \text { fixed } \\
-(-i)^{K} \sqrt{1-x} \frac{\exp \left\{2 \pi i s^{\prime} x\right\}-\exp \left\{2 \pi i\left(s^{\prime}-t^{\prime}\right\}\right.}{2 \pi i\left(s^{\prime}(1-x)-t^{\prime}\right)}, & s^{\prime}, t^{\prime} \text { fixed } \\
O\left(\frac{1}{M}\right) & \tilde{s}^{\prime}, t^{\prime} \text {; fixed } \\
O\left(\frac{1}{M}\right) & s^{\prime}, \tilde{t}^{\prime} \text { fixed }\end{cases}
\end{aligned}
$$

\footnotetext{
${ }^{6}$ In a situation where it is not, modification would be necessary.
} 
Looking back at eq. (2.14), we see that the overlap in a free fermion system is the exponential of a bilinear form in the coherent state parameters. with coefficients built from the matrices $D^{1}, D^{2}$. The simplification that the matrix elements, linking modes near $3 M / 4$ to modes near $M / 4$, vanish in the string limit means that there are no corresponding terms in the bilinear form. The absence of these terms means that the vertex conserves $\hat{P}$ as well as $Q$. Thus in the bosonized formulation the zero modes $a_{0}$ and $\tilde{a}_{0}$ are also separately conserved. We can call the modes near $3 M / 4$ right moving $(\hat{P}>0$ and those near $M / 4$ left moving $(\hat{P}<0)$. The decoupling also implies that the vertex factorizes into a factor involving only right moving modes times a factor involving only left moving modes.

As we have discussed each of the labels $s^{\prime}, r^{\prime}, t^{\prime}$, as well as the tilde labels, are integers plus a fractional part $\alpha(\tilde{\alpha})$ which depends on the charge $Q$ of the corresponding chain:

$$
\alpha=\left\{\begin{array}{ll}
\frac{1}{2} & Q \in 4 \mathbb{Z} \\
0 & Q \in 2+4 \mathbb{Z} \\
-\frac{1}{4} & Q \in 1+4 \mathbb{Z} \\
\frac{1}{4} & Q \in-1+4 \mathbb{Z}
\end{array}, \quad \tilde{\alpha}= \begin{cases}\frac{1}{2} & Q \in 4 \mathbb{Z} \\
0 & Q \in 2+4 \mathbb{Z} \\
\frac{1}{4} & Q \in 1+4 \mathbb{Z} \\
-\frac{1}{4} & Q \in-1+4 \mathbb{Z}\end{cases}\right.
$$

Note that the fractional parts of the right and left moving modes are the same when $Q$ is even, and they are opposites when $Q$ is odd.

Conservation of $Q$ implies selection rules for these fractional modes. Once the fractional modes are assigned to two of the chains, the third is fixed. The following transitions are allowed:

$$
\begin{aligned}
& (1 / 2,1 / 2) \rightarrow 1 / 2, \quad(1 / 2,0) \rightarrow 0, \quad(1 / 2,1 / 4) \rightarrow 1 / 4, \quad(1 / 2,-1 / 4) \rightarrow-1 / 4, \\
& (0,0) \rightarrow 1 / 2, \quad(0,1 / 4) \rightarrow-1 / 4, \quad(0,-1 / 4) \rightarrow 1 / 4, \\
& (1 / 4,1 / 4) \rightarrow 0, \quad(1 / 4,-1 / 4) \rightarrow 1 / 2, \quad(-1 / 4,-1 / 4) \rightarrow 0,
\end{aligned}
$$

where the fraction refers to the fractional part of the right moving modes. The selection rules involving only integer and half integer modes match those of the RNS spinning string model, with half odd integers the NS sector and integers the R sector. In fact, the overlap calculations in these sectors also coincide with those in the RNS formalism. The presence of quarter integer modes is a peculiar feature of the Heisenberg spin chain not found in the RNS string.

It is worth noting that these selection rules introduce a complication in manipulations with $D^{1}, D^{2}$, required to evaluate the full vertex, compared to some of the forbidden transitions. First notice that $r^{\prime}$ in the numerator of $D^{1}$ and $t^{\prime}$ in the numerator of $D^{2}$ can be replaced by their fractional parts. Then, for example, the forbidden transition $(0,0) \rightarrow 0$ would allow an overall factor of $1-e^{2 \pi i s^{\prime} x}$ to be removed from both $D^{1}$ and $D^{2}$. But for the allowed transitions, e.g. $(1 / 2,1 / 2) \rightarrow 1 / 2$ or $(0,0) \rightarrow 1 / 2$, one of these factors is $1-e^{2 \pi i s^{\prime} x}$ but the other is $1+e^{2 \pi i s^{\prime} x}$, so this simplification is not possible. 
To flesh this out a little, specialize to the case where the fractional parts of all modes are $1 / 2$. Then the matrix $D$ has elements

$$
\begin{aligned}
& D_{s r}^{1} \approx \begin{cases}-\sqrt{x} \frac{1+e^{2 \pi i \tilde{s}^{\prime} x}}{2 \pi i\left(\tilde{s}^{\prime} x-\tilde{r}^{\prime}\right)}, & \tilde{s}^{\prime}, \tilde{r}^{\prime} \text { fixed } \\
-\sqrt{x} \frac{1+e^{2 \pi i s^{\prime} x}}{2 \pi i\left(s^{\prime} x-r^{\prime}\right)}, & s^{\prime}, r^{\prime} \text { fixed } \\
O\left(\frac{1}{M}\right) & \tilde{s}^{\prime}, r^{\prime} \text { fixed } \\
O\left(\frac{1}{M}\right) & s^{\prime}, \tilde{r}^{\prime} \text { fixed }\end{cases} \\
& D_{s r}^{2} \approx \begin{cases}-i^{K} \sqrt{1-x} \frac{e^{2 \pi i \tilde{s}^{\prime} x}-1}{2 \pi i\left(\tilde{s}^{\prime}(1-x)-\tilde{t}^{\prime}\right)}, & \tilde{s}^{\prime}, \tilde{t}^{\prime} \text { fixed } \\
-(-i)^{K} \sqrt{1-x} \frac{e^{2 \pi i s^{\prime} x}-1}{2 \pi i\left(s^{\prime}(1-x)-t^{\prime}\right)}, & s^{\prime}, t^{\prime} \text { fixed } \\
O\left(\frac{1}{M}\right) & \tilde{s}^{\prime}, t^{\prime} \text {; fixed } \\
O\left(\frac{1}{M}\right) & s^{\prime}, \tilde{t}^{\prime} \text { fixed }\end{cases}
\end{aligned}
$$

Had $s^{\prime}$ and $\tilde{s}^{\prime}$ been integers instead of half odd integers the matrix $\left(D^{1} \mid D^{2}\right)$ could be written as a diagonal matrix with entries $\left(1+\exp \left\{2 \pi i \tilde{s}^{\prime} x\right\}\right)$ times a matrix with elements $1 /\left(s^{\prime} x-r^{\prime}\right)$ or $1 /\left(s^{\prime}(1-x)-t^{\prime}\right)$, which can be inverted by a method developed by J. Goldstone using analytic functions, or by the Neumann function methods of Mandelstam. When on shell methods were applied to the Neveu-Schwarz vertex by Hornfeck [30], he obtained a double sum over the simple closed form expressions for the bosonic string or Green-Schwarz three vertex. It is not mandatory that the answer be in closed form, but it is mandatory that the matrix $\left(D^{1} \mid D^{2}\right)$ reduce, in the limit $M \rightarrow \infty$ with $x=K / M$ fixed, to the overlap matrices used to derive the string scattering amplitudes, and they do [31].

On a more hopeful note, switching to bosonized language makes the overlap calculation treatable by Mandelstam's methods. And this can easily be extended to the conjectured $\Delta \neq 0$ case. The conjecture, supported by several explicit calculations, is that the only change for $\Delta \neq 0$ is a change in the radius of compactification [1,25]

$$
R_{\Delta}^{2}=\frac{1}{2 \pi T_{0}} \frac{\pi}{\mu}=\alpha^{\prime} \frac{\pi}{\mu}, \quad \Delta=-\cos \mu
$$

We see that the decompactification limit is $\Delta \rightarrow-1$. In this limit the chains with odd $M$ (odd $Q$ ) acquire infinite energy in the $R \rightarrow \infty$ limit and hence will decouple from finite energy processes. The conjectured equivalence to a compactified boson is very plausible in a neighborhood of $\Delta=0$. If it holds for all $\Delta$ in the critical range $-1<\Delta<1$, the scattering amplitudes of closed string theory will apply, including graviton graviton scattering in the decompactification limit.

\section{Acknowledgments}

I thank Lars Brink and Nathan Berkovits for helpful comments, and Sourav Raha for illuminating discussions. This work was supported in part by the Department of Energy under Grant No.DE-SC0010296. 
Open Access. This article is distributed under the terms of the Creative Commons Attribution License (CC-BY 4.0), which permits any use, distribution and reproduction in any medium, provided the original author(s) and source are credited.

\section{References}

[1] C.B. Thorn, Space from String Bits, JHEP 11 (2014) 110 [arXiv:1407.8144] [InSPIRE].

[2] R. Giles and C.B. Thorn, A Lattice Approach to String Theory, Phys. Rev. D 16 (1977) 366 [INSPIRE].

[3] C.B. Thorn, Reformulating string theory with the $1 / N$ expansion, in The First International A.D. Sakharov Conference on Physics, pp. 0447-454 (1991); published in Sakharov memorial lectures in physics, vol. 1, L.V. Keldysh and V.Ya. Fainberg eds., Nova Science Publishers, Commack, NY, pp. 447-453 (1992) [hep-th/9405069] [INSPIRE].

[4] F. Gliozzi, J. Scherk and D.I. Olive, Supersymmetry, Supergravity Theories and the Dual Spinor Model, Nucl. Phys. B 122 (1977) 253 [InSPIRE].

[5] P. Ramond, Dual Theory for Free Fermions, Phys. Rev. D 3 (1971) 2415 [InSPIRE].

[6] A. Neveu and J.H. Schwarz, Factorizable dual model of pions, Nucl. Phys. B 31 (1971) 86 [INSPIRE].

[7] A. Neveu, J.H. Schwarz and C.B. Thorn, Reformulation of the Dual Pion Model, Phys. Lett. $B 35$ (1971) 529 [INSPIRE].

[8] C.B. Thorn, Embryonic Dual Model for Pions and Fermions, Phys. Rev. D 4 (1971) 1112 [INSPIRE].

[9] A. Neveu and J.H. Schwarz, Quark Model of Dual Pions, Phys. Rev. D 4 (1971) 1109 [INSPIRE].

[10] G. 't Hooft, A Planar Diagram Theory for Strong Interactions, Nucl. Phys. B 72 (1974) 461 [INSPIRE].

[11] S. Sun and C.B. Thorn, Stable String Bit Models, Phys. Rev. D 89 (2014) 105002 [arXiv: 1402.7362] [INSPIRE].

[12] G. Chen and S. Sun, Numerical Study of the Simplest String Bit Model, Phys. Rev. D 93 (2016) 106004 [arXiv:1602.02166] [INSPIRE].

[13] S. Sun, Aspects of Stable String Bit Models, Ph.D. Thesis, University of Florida (2019).

[14] P. Goddard, C. Rebbi and C.B. Thorn, Lorentz covariance and the physical states in dual resonance models, Nuovo Cim. A 12 (1972) 425 [inSPIRE].

[15] P. Goddard, J. Goldstone, C. Rebbi and C.B. Thorn, Quantum dynamics of a massless relativistic string, Nucl. Phys. B 56 (1973) 109 [INSPIRE].

[16] O. Bergman and C.B. Thorn, String bit models for superstring, Phys. Rev. D 52 (1995) 5980 [hep-th/9506125] [INSPIRE].

[17] K. Bardakci and M.B. Halpern, New dual quark models, Phys. Rev. D 3 (1971) 2493 [INSPIRE].

[18] M.B. Green and J.H. Schwarz, Supersymmetrical Dual String Theory, Nucl. Phys. B 181 (1981) 502 [INSPIRE]. 
[19] C.B. Thorn, 1/N Perturbations in Superstring Bit Models, Phys. Rev. D 93 (2016) 066003 [arXiv: 1512.08439] [INSPIRE].

[20] C.B. Thorn, Protostring Scattering Amplitudes, Phys. Rev. D 94 (2016) 106009 [arXiv: 1607.04237] [INSPIRE].

[21] H.A. Bethe, Zur Theorie des Ferromagnetismus, Z. Phys. 61 (1930) 206.

[22] C.-N. Yang and C.P. Yang, One-dimensional chain of anisotropic spin spin interactions. 1. Proof of Bethe's hypothesis for ground state in a finite system, Phys. Rev. 150 (1966) 321 [INSPIRE].

[23] C.N. Yang and C.P. Yang, One-dimensional chain of anisotropic spin spin interactions. 2. Properties of the ground state energy per lattice site for an infinite system, Phys. Rev. 150 (1966) 327 [INSPIRE].

[24] E.H. Lieb, T. Schultz and D. Mattis, Two soluble models of an antiferromagnetic chain, Annals Phys. 16 (1961) 407 [INSPIRE].

[25] R. Giles, L.D. McLerran and C.B. Thorn, The string representation for a field theory with internal symmetry, Phys. Rev. D 17 (1978) 2058 [INSPIRE].

[26] S. Mandelstam, Interacting String Picture of Dual Resonance Models, Nucl. Phys. B 64 (1973) 205 [INSPIRE].

[27] S. Mandelstam, Interacting String Picture of the Neveu-Schwarz-Ramond Model, Nucl. Phys. B 69 (1974) 77 [INSPIRE].

[28] S. Mandelstam, Lorentz Properties of the Three-String Vertex, Nucl. Phys. B 83 (1974) 413 [INSPIRE].

[29] M.B. Green, J.H. Schwarz and L. Brink, Superfield Theory of Type II Superstrings, Nucl. Phys. B 219 (1983) 437 [INSPIRE].

[30] K. Hornfeck, Three Reggeon Light Cone Vertex of the Neveu-Schwarz String, Nucl. Phys. B 293 (1987) 189 [INSPIRE].

[31] N. Berkovits, Supersheet Functional Integration and the Interacting Neveu-Schwarz String, Nucl. Phys. B 304 (1988) 537 [INSPIRE]. 